\title{
The Role of Transoesophageal Echocardiography in Surgical Removal of a Mediastinal Tumour
}

\author{
Ajay Kumar Jha \\ Department of Anaesthesiology and Critical Care, Jawaharlal Institute of Postgraduate Medical Education and Research, Puducherry, India
}

DOI: https://doi.org/10.17925/HI.2020.14.2.118

$\mathrm{T}$ he complete and safe removal of a mediastinal mass requires cardiopulmonary bypass if the mass encroaches or compresses the heart and its great vessels. A 15-year-old male presented with a huge mediastinal mass with gradually worsening symptoms. Surgical removal of the tumour was planned under general anaesthesia and cardiopulmonary bypass based upon contrast-enhanced computed tomography of the chest, which suggested infiltration of the cardiac structures. Intraoperative transoesophageal echocardiography revealed a distinct separation of the tumour mass from the heart and its great vessels. The mass was excised en bloc without cardiopulmonary bypass.

\section{Keywords}

Transoesophageal echocardiography, mediastinal mass

Disclosure: Ajay Kumar Jha has no financial or non-financial relationships or activities to declare in relation to this article.

Review Process: Double-blind peer review.

Compliance with Ethics: Informed consent was received from the patient involved in this case study.

Authorship: The named authors meet the International Committee of Medical Journal Editors (ICMJE) criteria

for authorship of this manuscript, take responsibility

for the integrity of the work as a whole, and have given final approval for the version to be published.

Access: This article is freely accessible at

touchCARDIO.com (C) Touch Medical Media 2020.

Received: 12 April 2020

Accepted: 14 October 2020

Published Online: 30 October 2020

Citation: Heart International. 2020;14(2):118-20

Corresponding Author: Ajay Kumar Jha,

Department of Anaesthesiology and Critical

Care, Jawaharlal Institute of Postgraduate

Medical Education and Research, Puducherry

605006, India. E: drajaykjha@rediffmail.com

Support: No funding was received for

the publication of this article.
Perioperative management of a mediastinal tumour is an anaesthetic challenge. Echocardiography is not considered as a primary diagnostic modality in the evaluation of a mediastinal mass; however, it can provide valuable information in patients with clinical and diagnostic evidence of cardiac compression or encroachment. Judicious interpretation of perioperative transthoracic or transoesophageal echocardiographic images may be of great help to both the surgeon and anaesthesiologist in planning a proper management strategy. An effective preoperative plan and the preparation of femoral vessel cannulation for cardiopulmonary bypass initiation are imperative to avoid catastrophic respiratory and cardiac complications during anaesthetic induction. Furthermore, surgical manipulation may injure major vessels and the heart, and may induce non-sustainable haemodynamic alterations in case of the encasement of major vessels and the pericardium by the tumour.

\section{Case description}

A 15-year-old, $45 \mathrm{~kg}$ male was referred to our institution with a 2-month history of left-sided chest pain, difficulty in lifting the left arm, progressive dyspnoea, orthopnoea, recurrent cough and fever. Contrast-enhanced computed tomography (CECT) of the chest showed a heterogeneously enhancing anterior mediastinal mass with areas of chunky calcification and measuring $9.5 \mathrm{~cm}$ in its longest dimension. There was infiltration of the anterior chest wall, left atrium, left ventricle and descending thoracic aorta. Tracheobronchial anatomy was normal and there was no evidence of pleural or pericardial effusion. Furthermore, CECT did not suggest any evidence of pulmonary artery infiltration. Other diagnostic evaluations included a chest X-ray, which showed a cardiothoracic ratio of 0.7 and normal serum biochemical profiles. Needle-guided biopsy confirmed a malignant germ cell tumour. The patient was receiving chemotherapeutic drugs consisting of doxorubicin, etoposide and cisplatin, which had been initiated in the oncology department before referral to the cardiothoracic department. However, his symptoms gradually worsened. Surgical resection of the tumour was planned under cardiopulmonary bypass.

Induction of anaesthesia and endobronchial intubation with a double-lumen endotracheal tube were uneventful. Intraoperative transoesophageal echocardiography (TOE) examination revealed normal main pulmonary artery calibre without any sign of encroachment or compression (Figure 1). An outline of the left ventricle, left atrium and ascending aorta was clearly visible and there was no evidence of compression of the left ventricular outflow tract or the ascending aorta (Figure 2). There was no evidence of excessive blood flow acceleration in the pulmonary artery or the ascending aorta. The pressure gradient across the aortic and the pulmonary valve was minimal. Three-dimensional TOE revealed a distinct separation between the tumour mass and the heart (Figure 3).

The surgical plan was changed after interpretation of the TOE images and manual palpation of the tumour. Left-lung isolation was achieved to facilitate surgical resection and to avoid damage to the lung parenchyma. The tumour was resected en bloc with a clean margin, and without 
Figure 1: Upper oesophageal view showing normal pulmonary artery calibre without evidence of compression by the mediastinal mass

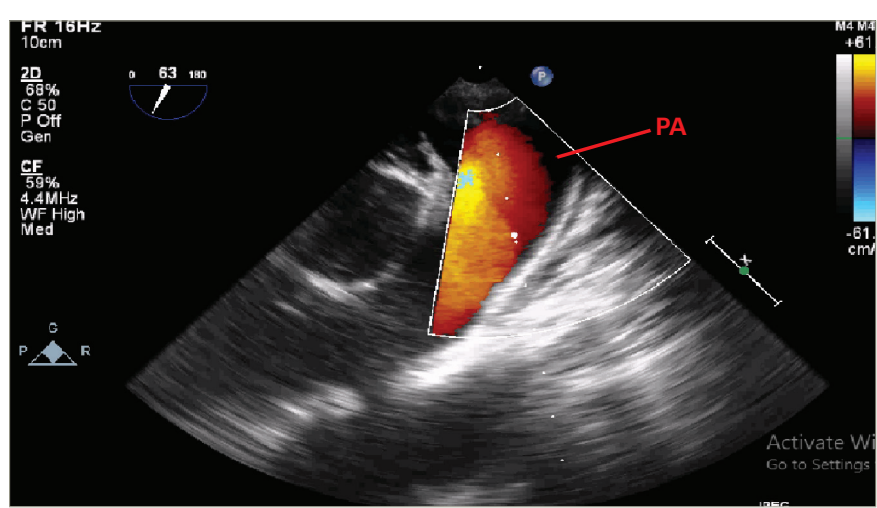

$P A=$ pulmonary artery.

Figure 2: Two-dimensional echocardiography in deep transgastric view
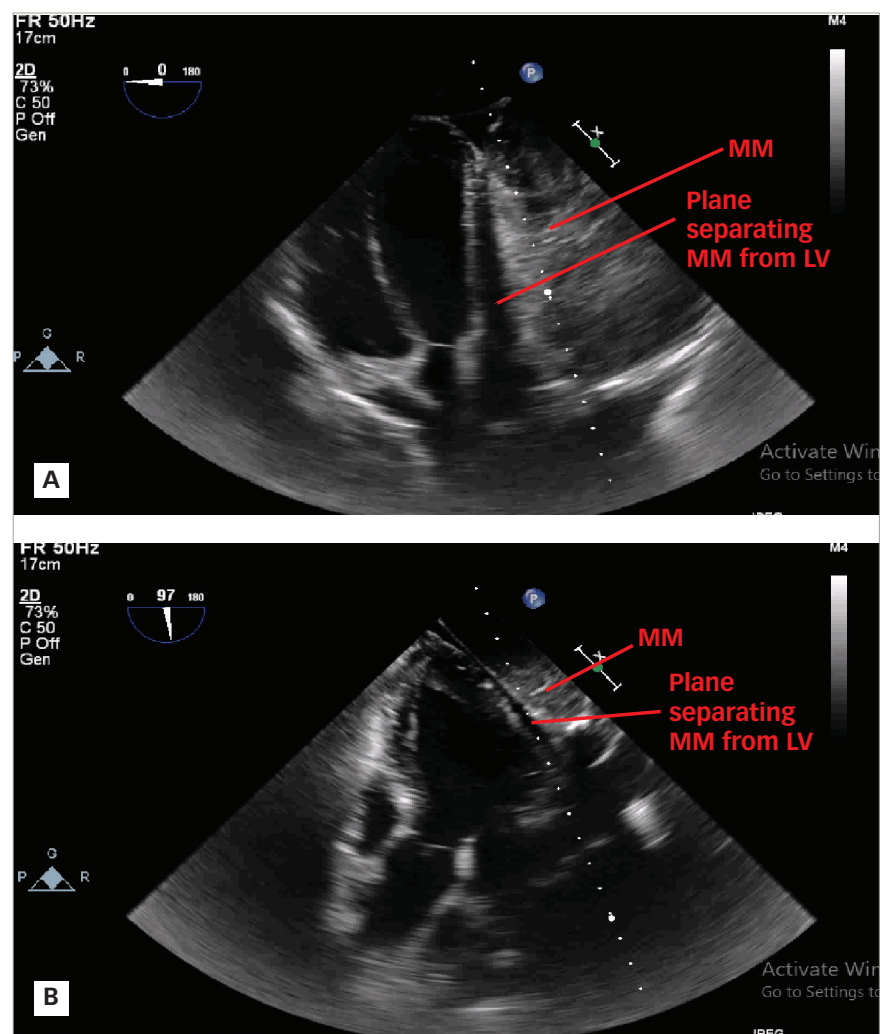

Separation of the mediastinal mass (MM) from the left ventricle (LV) and ascending aorta at 0 degrees (A) and 97 degrees (B)

cardiopulmonary bypass, through hemiclamshell thoracotomy (Figure 4). Noradrenaline infusion at $0.05 \mu \mathrm{g} / \mathrm{kg} / \mathrm{min}$, and intermittent boluses of phenylephrine, were used to treat hypotension during handling of the tumour and the heart. The post-operative period was uneventful and the patient was extubated after 4 hours. The following postoperative day, the patient was moved to the oncology department for chemotherapy and follow-up. The patient's consent was obtained for the publication of this article during follow-up.

\section{Discussion}

Five to $10 \%$ of all germ cell cancers arise in extragonadal locations, such as a typical midline structure, particularly in the mediastinum.
Figure 3: Three dimensional echocardiography of the mediastinal mass
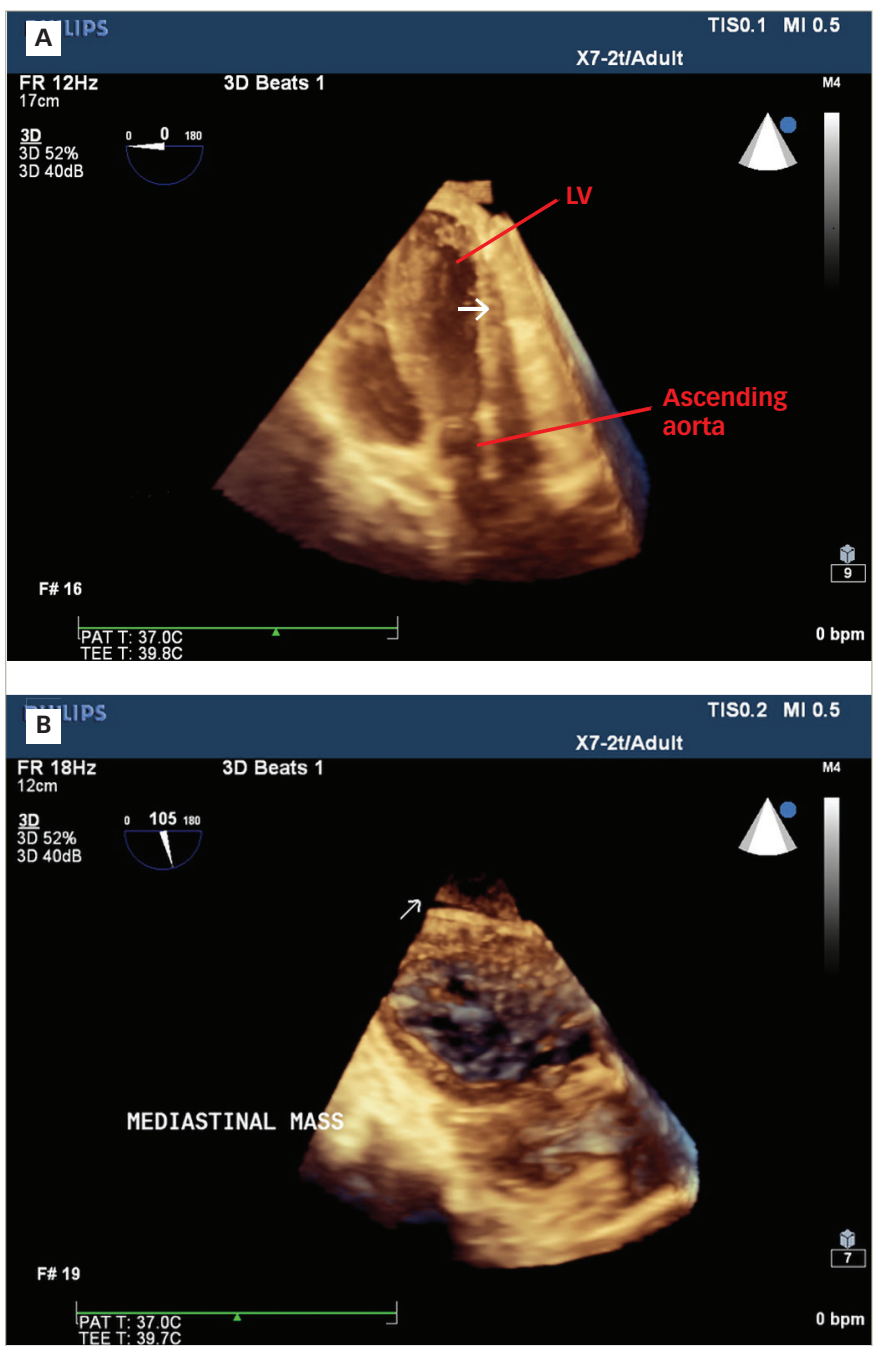

Distinct separation of the mediastinal mass from the left ventricle (LV) and ascending aorta shown by deep transgastric view at 0 degrees $(A)$ and basal transgastric view at 105 degrees (B).

Figure 4: Resected en bloc mediastinal mass

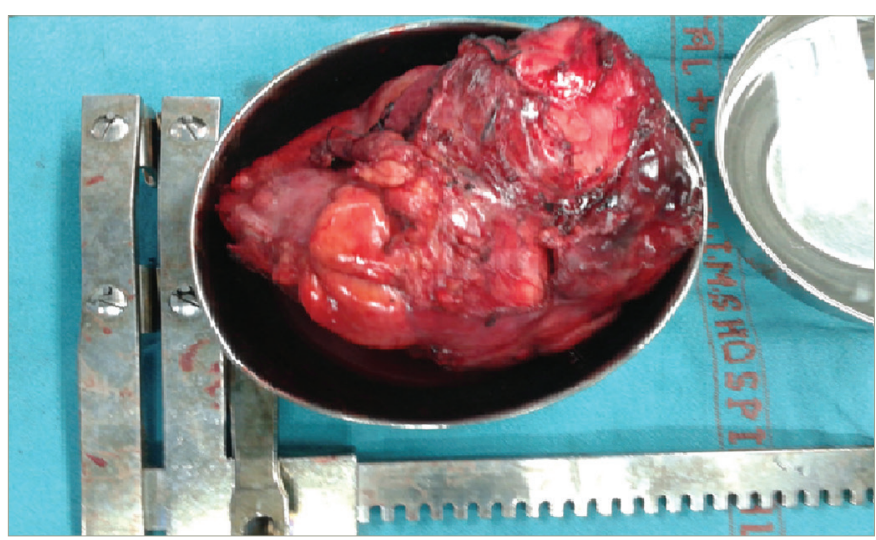

There is no potential space within the chest cavity, and any space-occupying lesion tends to displace other vital structures. A mass within the mediastinum can encroach and/or compress the heart and its great vessels. Encroachment does not usually produce haemodynamic changes, but compression by the mass produces haemodynamic

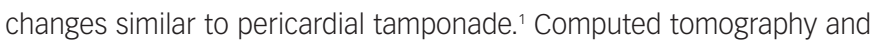


magnetic resonance imaging are essential for complete staging, and assessment of extent and surgical resectability of the mass.

TOE can provide supplemental information regarding encroachment and compression. TOE is more accurate than transthoracic echocardiography in detecting mediastinal masses (90\% versus $73 \%$ ), in identifying their structure (100\% versus 90\%), and in evaluating their relationship with contiguous organs (89\% versus $81 \%$ ). ${ }^{2}$ TOE has its limitations; it cannot delineate every surface and border of the heart. However, real-time images may provide evidence of mediastinal pleural sliding, which may help to exclude tumour invasion to the surrounding structures. Furthermore, real-time TOE imaging during surgical manipulation helps to alert the surgeon during manipulation and guides the anaesthesiologist in optimising the haemodynamics. We tried to visualise the mass in all possible TOE views. It was only possible to visualise separation of the mediastinal mass from the cardiac structures in the deep transgastric view.

The difference in the CECT report and TOE interpretation could be due to chemotherapy-induced shrinkage in the tumour size; however, CECT was only performed 6 days prior to surgery and the patient's symptoms further worsened during those intervening days. We are of opinion that the radiographer may have over-read the CECT chest images. The decision not to use the cardiopulmonary bypass from the beginning was not solely based upon TOE images. TOE gave additional information about the separation of the mediastinal mass from the heart, and the surgeon further confirmed this separation by manual palpation.

Despite haemodynamic fluctuations, the patient tolerated surgical manipulation of the heart without major haemodynamic compromise. Had there been major haemodynamic deterioration, we would have used cardiopulmonary bypass. Complete isolation and en bloc resection of tumours are difficult without cardiopulmonary bypass. It is equally challenging for the anaesthesiologist to maintain normal haemodynamic parameters during manipulation of the heart. Discernible tissue planes should be present for complete isolation and en bloc resection of a tumour. Surgical resection of malignant germ cell tumours after chemotherapy has shown favourable long-term survival. Complete resection should be performed to achieve long-term survival. ${ }^{3}$

\section{Conclusion}

TOE can provide valuable supplemental information about the extent of encroachment or compression by a mediastinal tumour. TOE can continuously estimate the ventricular end diastolic volumes and pressures, and thus helps with intraoperative haemodynamic management during tumour mobilisation and resection. $\square$

1. D'Cruz IA, Feghali N, Gross CM. Echocardiographic manifestations of mediastinal masses compressing or encroaching on the heart. Echocardiography. 1994;11:523-33.
2. Faletra F, Ravini M, Moreo A. Transesophageal echocardiography in the evaluation of mediastinal masses. J Am Soc Echocardiogr. 1992;5:178-86.

3. Kang CH, Kim YT, Jheon SH. Surgical treatment of malignant mediastinal nonseminomatous germ cell tumor. Ann Thorac Surg. 2008;85:379-84. 